\section{Young people leaving the UK armed forces at increased risk of suicide}

\section{QUESTION}

Question: What is the rate of suicide, timing of suicide, risk factors for suicide and rate of contact with mental health services among individuals leaving the UK armed forces?

People: 233803 individuals leaving the British Army (59\%), Naval Service (22\%) or Royal Air Force (20\%) between 1 April 1996 and 31 December 2005. Median age was 25 years (2034 years) and $90 \%$ were male. Data were compiled by the Defence Analytical Services Agency (DASA). Reservists and people serving in the Gurkha regiment were excluded.

Setting: Population study, UK; April 1996-December 2005.

Risk factors: The database of those who had left the armed service was linked with suicide data. Survival analyses using Cox's proportional hazards models were calculated to assess the effects of age and length of service on suicide rate. The effect of gender, rank, training, marital service and length of service on suicide rate were also assessed. Rate of contact with the mental health services was determined from The Inquiry patient database. A case control analysis was undertaken on this group in contact with mental health services with five controls from the general population matched in age, gender and year of death to describe the characteristics of this group.

Outcomes: Coroner's verdict of suicide or undetermined death (open verdict). Suicide data were collected from the National Confidential Inquiry on Suicide and Homicide by People with Mental Illness (The Inquiry) databases. These suicide data were linked with the DASA data to determine the suicide rate among people discharged from the services. The overall standardised mortality ratio (SMR) was calculated, in addition to age specific mortality ratios using 5 year age bands so that suicide rates could be compared with the general population.

\section{METHODS}

Design: Retrospective cohort study.

Follow-up period: Up to 8 years, 9 months; mean follow-up 5 years.

\section{MAIN RESULTS}

Overall, 224 people died by suicide after leaving the armed forces. Median age was 22 years (19-29 years), 215 (96\%) were male, $163(73 \%)$ had served in the Army, 34 (15\%) in the Naval services and $27(12 \%)$ in the Royal Air Force. Median time to death after discharge was 21 months (1657 months). For men, the overall suicide rate was not greater than in the general population (SMR 96.5, 95\% confidence interval (CI) 84.4 to 110.4; see webextra table 1). Compared with the general population, risk of suicide was higher in men aged 16-19 years (age specific mortality ratio 292.9, 95\% CI 185.1 to 441.1 ) and 20-24 years (age specific mortality ratio $169.9,95 \%$ CI 134.0 to 212.7 ). Risk of suicide was lower than the general population in people aged 3059 years (see webextra table 2). For women, the overall suicide rate was not greater than in the general population. Risk of suicide was greatest in the first 2 years compared with discharge longer than 2 years (suicide rate per 100000 person years: $24.7,95 \%$ CI 20.1 to 30.2 vs $18.7,95 \%$ CI 15.7 to 22.4). Suicide risk was associated with male gender, younger age at discharge, being unmarried, lower rank, being untrained and length of service less than 4 years (see webextra table 2). Fewer people from the armed forces contacted mental health services in the year before death compared with the general population $(n=47,21 \%, 95 \%$ CI 16 to $27 \%$ vs $28 \%, 95 \%$ CI 27.5 to $28.2 \%$ ).

\section{CONCLUSIONS}

Men aged under 25 years who leave the UK armed forces are at increased risk of suicide. These risks are greatest in the first 2 years after discharge.

\section{ABSTRACTED FROM}

Kapur N, While D, Blatchley N, et al. Suicide after leaving the UK armed forces-a cohort study. PLoS Med 2009;6:e26.

Correspondence to: Navneet Kapur, Centre for Suicide Prevention, University of Manchester, Manchester, UK; nav.kapur@manchester.ac.uk

Source of funding: Veterans Project Unit, UK Ministry of Defence.

- Tables 1 and 2 are published online only at http://ebmh.bmj.com/content/vol12/ issue4
S uicide among UK Armed Forces personnel receives much political, public and media attention. Suicide statistics comparing serving personnel with the general population are published annually. However, there are no studies which have examined the risk of suicide among UK veterans (those who have left the Armed Forces). Veterans may be at particular risk of suicide, given that a minority of those who leave the Armed Forces find the transition back into "civilian" life difficult. ${ }^{1}$

Kapur and colleagues have linked a database containing details of 233803 individuals who have left the regular UK Armed Forces (between 1 April 1996 and 31 December 2005) to the databases held by the National Inquiry into Suicide and Homicide. This linkage led to the identification of 224 suicides among veterans. Standardised mortality ratios (based on data from the UK general population) showed that the number of suicides among male veterans was no different from the general population. Age group specific rate ratios showed that for all age groups the rate of suicide was lower for veterans compared with the general population, with the exception of young males $(<25$ years) where the rate ratios were significantly raised. Risk factors for suicide were being young ( $<25$ years), male, untrained, in the Army, holding a non-officer rank, not being married and having short length of service $(<4$ years).

These results are similar to those reported among serving personnel'; serving personnel have fewer suicides than expected (compared with the UK general population) but there are more suicides than expected among those under 20 years of age in the Army. It is likely that pre-service vulnerability ${ }^{3}$ may explain much of the excess suicide "risk" among young military personnel (regardless of serving status).

Nicola T Fear, DPhil and Simon Wessely, MD, FRCP, FRCPsych, FMedSci

Institute of Psychiatry, King's College London, London, UK

Competing interests: SW is Honorary Civilian Consultant Advisor to the British Army.

1. Iversen A, Dyson C, Smith N, et al. 'Goodbye and good luck': the mental health needs and treatment experiences of British ex-service personnel. $\mathrm{Br} J$ Psychiatry 2005;186:480-6.

2. Fear NT, Ward VR, Harrison K, et al. Suicide among male regular UK Armed Forces personnel, 1984-2007. Occup Environ Med 2009:66:438-41.

3. Iversen AC, Fear NT, Simonoff E, et al. Influence of childhood adversity on health among male UK military personnel. Br J Psychiatry 2007;191:506-11. 